Eberhard H. Lehmann, Anders Kaestner, Christian Grünzweig, Dadid Mannes, Peter Vontobel, Steven Peetermans

Neutron Imaging \& Activation Group, Paul Scherrer Institut, Villigen, Switzerland

\title{
Materials research and non-destructive testing using neutron tomography methods
}

This paper describes the modern tool of neutron tomography as an alternative and complement to the more common $\mathrm{X}$-ray options and its potential for applications in materials research and engineering. Based on our own practical experiences, the challenges, success and limitations of neutron tomography are sketched and the potential for further improvements and methodical extensions is described. Using the performance at dedicated neutron imaging beam lines, there are many new aspects which only can be made available with neutron methods such as energy-selective imaging near Bragg edges, imaging with polarized neutrons and even imaging in the resonance region. Therefore, it is of high importance to get more and easier access to suitable beam ports at advanced neutron sources to make methodical progress and to attract more scientific and industrial users.

Keywords: Materials research; Neutron tomography; Neutron diffraction; Phase contrast; Beam attenuation

\section{Introduction}

With the availability of digital neutron imaging detection systems, the access to suitable neutron beam ports, and the implementation of required computation power, it became possible to establish neutron tomography as a research tool in several user labs [1-3] and to perform investigations on a routine basis. It has now moved beyond proof-of-principle experiments to play an essential role in the neutron source user programs with high impact to materials research studies.

Most of the existing setups are based on camera detection systems using a neutron sensitive scintillation screen as the primary sensor [4]. Systems with amorphous Si flat panels are also used successfully, but they are limited by their fixed pixel size and field-of-view. A promising new trend is the use of pixilated detectors using a micro-channel-plate sensor (doped with a neutron absorber) [5] due to its high detection efficiency and flexible acquisition and read-out characteristics.

Neutron tomography is a three-dimensional "extension" of neutron radiography. Even though tomography is a standard method for neutron imaging, there is still an on-going development to overcome limitations of the traditional computed tomography using a sufficient number of wellexposed projections. Within this report we will describe the state-of-the-art of neutron imaging and give an outlook towards further methodical improvements and applications.
As in X-ray tomography, neutron tomography is based on the beam attenuation by the object under investigation and the observation of the resulting intensities $I$ from a number of possible viewing directions in comparison to the initial intensity $I_{0}$. By means of the inverse Beer-Lambert's law (for one projection in radiography mode):

$\Sigma \cdot d=\ln \left(\frac{I_{0}}{I}\right)$

and by knowing sample thickness $d$ the material specific attenuation coefficient $\Sigma$ can be derived. Having several different materials in the beam it is more correct to use the line integral along the viewing direction:

$\int \Sigma(x, y) \cdot \mathrm{d} s=\ln \left(\frac{I_{0}}{I}\right)$

The whole procedure in practical neutron tomography is realized as the filtered back-projection procedure [6], mostly using the parallel beam approximation. The obtained three-dimensional voxel matrix $\Sigma(x, y, z)$ describes the object and can be used to perform segmentation of different areas in a qualitative and even quantitative manner.

Tools for the reconstruction step are available on a commercial basis (e.g. [7]) or as in-house developments (e.g. [8]). The resulting stacks of horizontal slices $\Sigma(x, y)$ can be taken as input data for visualization tools (e.g. $[9,10])$. Some results of data analysis using these tools are given below.

\section{Neutrons vs. X-rays}

From the historical point of view, the neutron methods have a delay of decades and years until now in comparison to the $\mathrm{X}$-ray technologies. Here, we summarize some topics and milestones for similar achievements:

- free neutrons were discovered 37 years after the X-rays were found

- neutron imaging started 50 years after first X-ray images were made

- neutron diffraction comes 30 years later than X-ray diffraction

- neutron tomography comes 25 years later than X-ray tomography in hospitals

- phase contrast imaging with neutrons comes 10 years later than with X-rays

- neutron imaging is now a competitive and complementary method compared to the X-ray techniques 
However, latest developments of X-ray sources (synchrotron light sources, free-electron lasers) give further potential for improvements in the X-ray world, where neutrons can probably take profit later too. It has been reported [11] that since 1990 the source brightness at synchrotrons has been increased by three orders of magnitude while over the same period radiation focusing resolution (e.g. by zone plate devices) has been improved by more than two orders of magnitude. This progress is mainly pushing highest spatial resolution and ultra-fast acquisition forward.

In this perspective the question of neutron beam intensity remains a major issue. Even with the strongest neutron sources (e.g. the ILL reactor Grenoble) the beam intensities cannot be higher that about $10^{10}$ neutrons $\mathrm{cm}^{-2} \mathrm{~s}^{-1}$, which is by orders of magnitude less than at synchrotrons (e.g. $10^{18}$ photons s $\mathrm{mm}^{-2} \mathrm{mrad}^{-2} 0.1 \% \mathrm{BW}^{-1}$ at SLS, PSI). The beam intensity can be used either to increase the acquisition rate, the energy bandwidth or the divergence or to increase the coherence. Therefore, a careful decision has to be made as to which parameter is to be optimized in the measurement.

Generally, thermal and cold neutrons are preferred in neutron imaging due to their efficient detectability and the high variation in the attenuation properties of the sample materials.

From their interaction mechanism neutron imaging differs from X-rays in principle since neutrons interact with the atomic nuclei while X-rays interfere with the electrons in the shell. Therefore, light elements are transparent for $\mathrm{X}$-rays and heavy elements get more and more opaque with higher mass. Neutron interaction with matter (in terms of the cross-section data vs. material number) is not as systematic as in the case of X-rays but generally holds: light elements interact strongly either by scattering $(\mathrm{H}, \mathrm{C})$ or by absorption (B-10, Li-6) and many heavy elements (such as $\mathrm{Pb}, \mathrm{Bi}, \mathrm{U}$ ) are more transparent for neutrons (see the example of a study with X-rays and neutrons in Fig. 1).

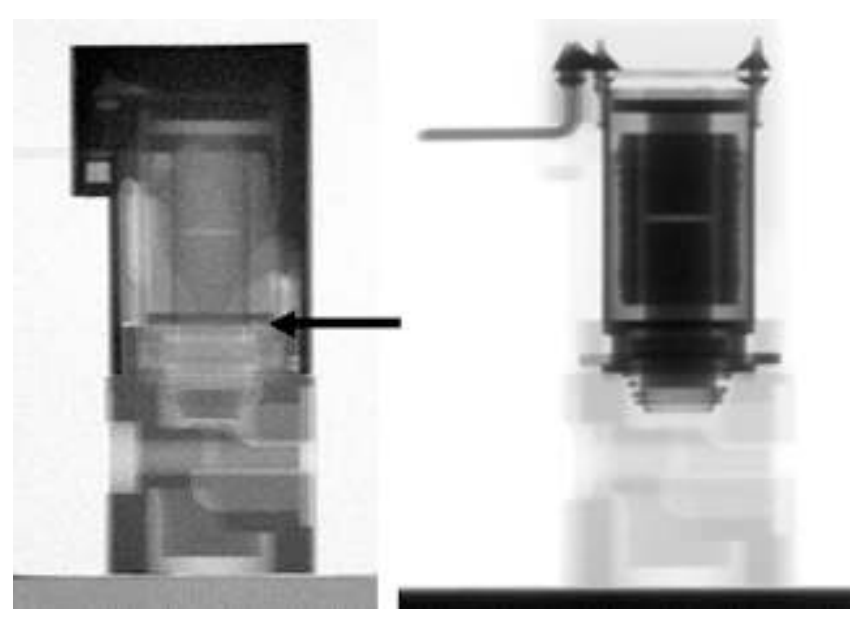

Fig. 1. Transmission images with thermal neutrons (left) and $100 \mathrm{kV}$ X-rays (right) of a plastic sealed sensor demonstrate the differences in the two methods: metals are transparent for neutrons and plastic gives high contrast; for X-rays the situation is reversed. The advantage of the image data is the visibility of the O-ring in the middle of the device (see the arrow).

\section{Neutron tomography on the macro-scale}

The high penetrability of many metals with neutrons gives reason to perform neutron tomography with samples of some $\mathrm{cm}$ in thickness. In particular objects of aluminum, magnesium, copper, brass, lead, bismuth and even steel are preferably studied using neutrons. Neutrons can mostly penetrate larger sample dimensions than X-rays. This is demonstrated by a wedge study (e.g. for $\mathrm{Al}$ - see Fig. 2 right), which shows that beam hardening effects have often much less relevance for neutrons than for high-energy X-rays.

This makes it possible to analyze entire cylinder heads of combustion motors in three dimensions with high precision. The only limitations are the spatial resolution of the detector and the dimensions of the beam. A major advantage in such cases is the visibility of organic material (oil, lubricants, adhesives, fuel) inside or behind the metallic structures.

The two beam lines at PSI (NEUTRA [12] for thermal neutrons and ICON [13] for cold neutrons) provide beam diameters of $30 \mathrm{~cm}$ and more and the corresponding detectors accepting such large beam dimensions. This is the "MAXI" option marked in Fig. 3. For even larger samples scanning in one or two directions is possible. This has already been done in several cases. Some applications are from industry (large ceramic insulators, complete engines) but also from cultural heritage studies (bronze sculptures [14], old weapons [15], block excavations).

\section{Neutron tomography on the micro-scale}

Challenged by the spatial resolution that can be obtained with X-ray tomography, which is much below one micrometer, there are also demands and intensions to get the highest possible resolution in neutron imaging. Whereas the intensity in synchrotrons enables sorting photons in a manner to set an ideally parallel beam, the magnification option from a very narrow source point is used in X-ray tube setups forming a cone beam.

Neither of these alternatives is possible for neutrons. First, neutron sources with reasonable intensities cannot be built for heat removal reasons and real extended sources have their inherent divergence. Furthermore, the neutron detection reaction has its own inherent "blurring" as neutrons have to be converted first to charged particles before a measureable excitation (e.g. scintillation, ionization) is induced.

Nevertheless, it was possible to build a setup [16] with a pixel-size of $0.0135 \mathrm{~mm}$ and $27.5 \mathrm{~mm}$ field-of-view (MICRO in Fig. 3). This detection system has been used for many tomography studies at the cold neutron imaging beam line ICON [13] at the spallation neutron source SINQ [17]. In particular, organic materials deliver a high contrast due to the interaction of the neutrons with hydrogen even in metallic sealing (see Fig. 4).

As indicated in Fig. 3, various tomography systems can be made available when the optical system of the setup is tuned in the right manner. In addition to the camera-lens system the scintillation screen plays an important role. While for large extended samples a $0.3 \mathrm{~mm}$ thick scintillation screen with high conversion efficiency can be accepted because the pixel size is in the same order we need very thin screens for highest spatial resolution. The usage of Gadox 
screens with only $0.01 \mathrm{~mm}$ thickness has been found most suitable.

Based on the positive experience with the MICRO setup, we initiated the "neutron microscope" project with the similar approach: a high-sensitivity camera system with a high number of small pixels combined with a perfect and highly efficient and magnifying lens $(1: 5)$. The system will use specially designed scintillators of $1 \mathrm{~cm}^{2}$ size with microstructure (nano-particles, columns), which are still under development. Optionally, neutron optical elements will be used to increase the intensity on the sample.

The higher spatial resolution is required to fill the resolution gap to the X-ray alternatives (from $\mathrm{mm}$ to micro-meters) while keeping in mind the high contrast for hydrogen, lithium and boron as contrast features. In this manner, soil physics, plant physiology, electrochemistry and other research fields will profit from a successful installation.
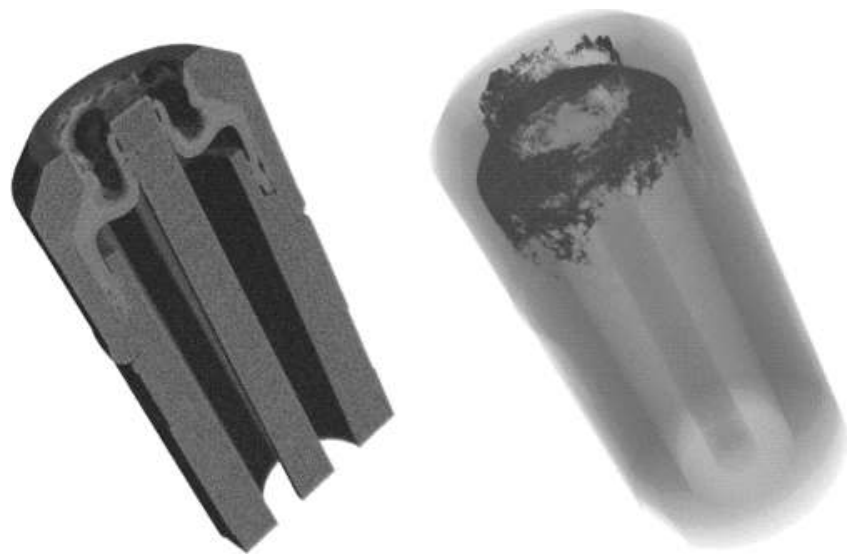

Fig. 4. Visualizations of combustion residuals at the head of a pressure sensor (outer diameter $6.7 \mathrm{~mm}$ ): left - volumetric slice; right - 3D distribution of the organic material at the steel cladding which is set to "transparent".
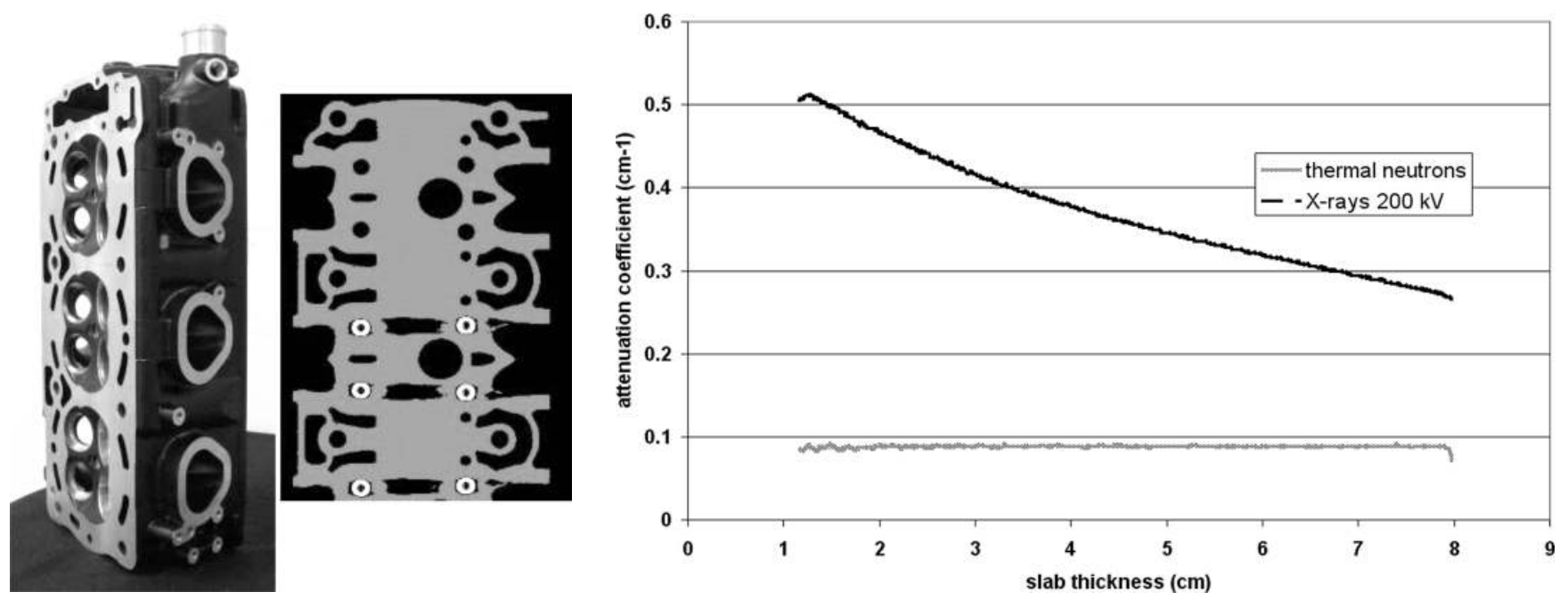

Fig. 2. Image of a 3-cylinder head of a combustion engine; left - photo, middle - slice of the upper part in the center: no distortion by beam hardening is visible. Right: attenuation coefficient data in dependence of sample thickness - in the case of $\mathrm{Al}$ there is no effect of beam hardening as happens in the case of $200 \mathrm{kV}$ X-rays. A beam hardening correction, common in X-ray studies, can be avoided in the neutron imaging case.

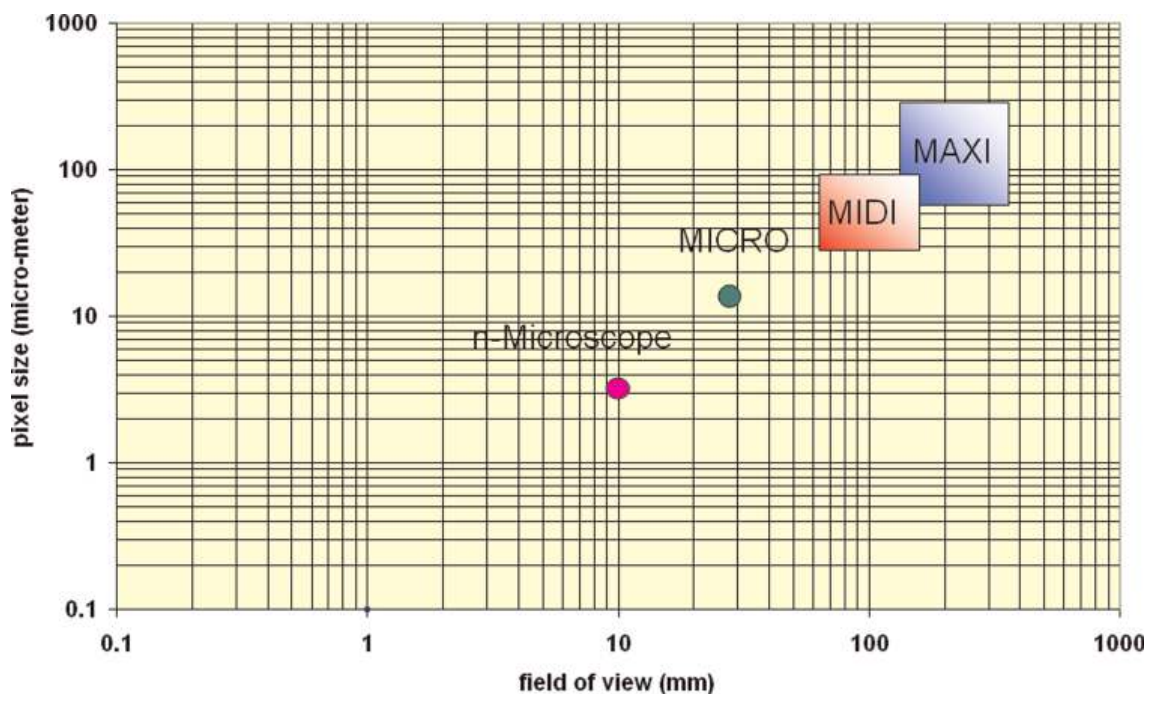

Fig. 3. Different tomography systems available at PSI and their working range in respect to the field-of-view and the pixel size. The "neutron microscope" is still under development. 


\section{Energy-selective tomography}

Most routine neutron tomography studies are performed with "white" beams of either cold or thermal neutrons. The corresponding attenuation coefficients $\Sigma_{\text {eff }}$ represent averaged values as:

$\Sigma_{\text {eff }}=\frac{\int \Sigma(E) \cdot \varepsilon(E) \cdot \varphi(E) \mathrm{d} E}{\int \varepsilon(E) \cdot \varphi(E) \mathrm{d} E}$

For materials with a simple cross-section to wavelength relationship (such as $1 / v$ absorbers $-v$ is the neutron velocity) these averaged values can easily be used for a precise quantification as the neutron spectrum $\phi(E)$ and the detection efficiency $\varepsilon$ are well known and fixed for all investigations.

In the case of crystalline structural materials (e.g. Fe, $\mathrm{Ni}$, $\mathrm{Cu}, \mathrm{Zr}, \mathrm{Pb}, \mathrm{Al}, \ldots)$ the elastic neutron scattering as reflections at the lattice planes of the crystallites form the crosssection data as shown in Fig. 5. This behavior can be described in a simplified manner (powder approximation) by a superposition of scattering functions:

$\sigma_{\mathrm{coh}}^{\mathrm{el}}(\lambda)=\frac{\lambda^{2}}{2 V_{0}} \sum_{d_{\vec{h}}=0}^{2 d_{\vec{h}}<\lambda}\left|F_{\vec{h}}\right|^{2} d_{\vec{h}}$

with the wavelength $\lambda$, the lattice spacing vector $h$ of the corresponding plane and the structure factor $F$. More details can be found, e.g., in [18].

Using narrow bands from the neutron spectrum now enables investigation of the crystalline structural behavior in the transmission mode since only lattice planes can provide scattering, which provide contributions according to Bragg's law (see Eq. (6)) in that wavelength range.

In practice, the required energy-resolution is obtained either through time-of-flight measurement at pulsed neutron sources or by using a mono-chromatizer (e.g. a mechanical velocity selector or a double crystal mono-chromator) at continuous sources.

Such investigations are of most relevance close to the socalled Bragg edges, they are prompt jumps in the cross-sec-

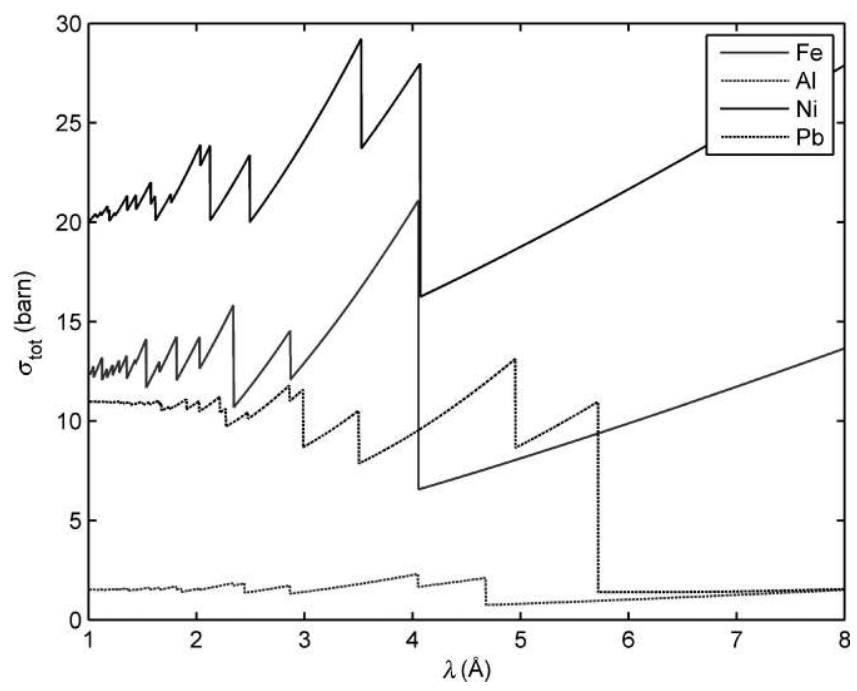

Fig. 5. Energy selective studies with a rolled Al plate in transmission radiography mode (above) at different wavelengths and a view of tomographic data at $4.0 \AA$ (wavelength spread $15 \%$ ). tions. Their position is related to crystallographic phase and strain, and their shape can strongly be influenced by presence of texture. Imaging around such a phase-specific Bragg edge thus provides spatial information on crystal phase distribution within the sample [19, 20].

In the case of samples with few and large grains (i.e. above the detector resolution) in the beam direction, the powder approximation is no longer valid and no clear Bragg edges can be observed anymore. Rather, spatially non-uniform contrast is exhibited by the sample, as grains that are so oriented within the beam that they fulfill Bragg's law will diffract neutrons out of the direct beam as opposed to neighboring grains which are oriented differently and do not.

We investigated two classes of materials in energy-selective mode: stainless steel and rolled Al plate sheets which both have structures with grain size above the resolution of the detection systems in the order of some tenths of $\mathrm{mm}$. In both cases, the white beam images and those obtained at wavelength above the last Bragg edge (absorption range) do not show any internal micro-structures [21]. In the first case the averaging over all energies blurs out the features from micro-crystallites and in the second case there are no scattering effects induced anymore.

Under tomography conditions, such samples should fail in principle because the attenuation is not always in correspondence to Eq. (1) because the out-scattering is determined by the crystalline structure and only for a limited angular range. However, reconstruction using filtered back projection provides a reasonable qualitative behavior as shown for the rolled Al plate in Fig. 6.

Future installations at pulsed sources will provide better conditions than we have so far had available. In particular the time-of-flight mode can provide a much higher energy resolution. Some facilities are already under realization [22-24] while other projects have been initiated [25, 26].

\section{Time-dependent tomography}

Many neutron-imaging applications involve the study of dynamic processes. Due to sample heterogeneity these sam-

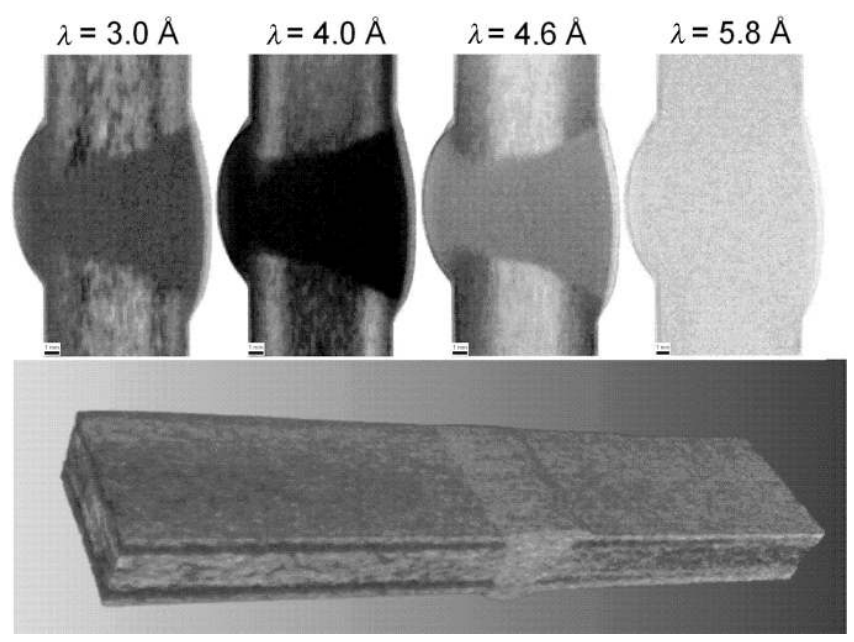

Fig. 6. Energy selective studies with a rolled Al plate in transmission radiography mode (above) at different wavelength and a view of tomographic data at $4.0 \AA$ (wavelength spread $15 \%$ ). 
ples are often studied using tomography to provide three-dimensional information about the process movements. This puts real-time constraints on the CT acquisition. The acquisition rate of complete projection data sets must be faster than the intrinsic motion in the sample; otherwise motion artifacts will appear and corrupt the image data. In some cases, it is also difficult to define the ideal acquisition rate.

As described in [27] for the case of the water migration process in lightweight aggregate (LWA) and superabsorbent polymer (SAP) particles, a specific acquisition scheme can be used in order to optimize between motion blurring, time resolution and quantitative accuracy. One approach to arranging the projection angle most efficiently is the Golden ratio: $\varphi=(1+\sqrt{5}) / 2$, where the acquisition angle of the $i$-th projection is defined according to:

$\theta_{\mathrm{i}}=i \varphi \pi \bmod \pi$

The result of Eq. (5) is that two consequent projections in time are nearly orthogonally separated in rotation. By rotating the sample in this manner, every chronological contiguous subsequence of the projection data represents a complete coverage of the scan $\operatorname{arc}(\pi$ or $2 \pi)$. This is an important feature for the ability to reconstruct the data with different spatiotemporal resolutions. This scheme can be started with no predefined end and can arbitrarily be terminated at any convenient end point.

\section{Diffractive neutron imaging}

As described in Section 5, the neutron attenuation in transmission imaging is predominantly triggered by scattering processes for most of the sample materials in the range of thermal and cold neutron energies. Some exceptions are the strong absorbers such as B-10, Cd or Gd.

The transmitted beam fraction is registered in standard setups as the two-dimensional shadow image of the sample while the scattered component is often ignored or considered as misleading because the quantification accuracy is affected.

However, the scattered neutron faction can also be of high importance for the characterization of the sample material. This "business" of the whole neutron scattering community is to analyze the scattered neutrons under welldefined and well tuned conditions (energy selection, collimation, sample orientation, beam polarization, special sample environments ...) - to obtain information on the atomic length scale.

Taking the well-known Bragg's law, Eq. (6), as a guideline, the lattice plane distances $d$ and their orientation can provide a strong signal as reflection into determined direction $\theta$ if the wavelength $\lambda$ is suitable.

$2 d_{h k l} \sin \theta=\lambda$

In single-crystalline samples, characteristic Laue patterns occur which can be used to describe the sample's properties in respect of the orientation and maybe the crystal size.

With the imaging approach and the capability of the used 2D detection systems, taking into account the fully homogeneous illumination of even extended samples (tens $\mathrm{cm}^{2}$ ) a second detector system aligned aside the beam direction allows investigating the scattered component in great detail. These measurements can be done either with a white beam to excite simultaneously all lattice planes or for selected narrow wavelengths bands. The so called "double detector setup" is sketched in Fig. 7 as a combination of two independent imaging devices with different field-of-view, resolution and timing.

We used this approach to describe the global crystalline structure of engineering materials such as single crystalline components used in turbine plates and other samples of large grained single crystals.

Comparing the transmission image with the diffracted image from the example of the "single crystal" turbine blade (Fig. 7) clearly indicates no visible inhomogeneity of the bulk of the object, but a structure in the diffracted image caused by slightly $\left(<1^{\circ}\right)$ disoriented areas. In this manner, this technique is unique for global characterization of how the different crystals are arranged and aligned in a macroscopic sample.

For the moment, these studies are focused on the radiography mode for reasons of relatively long acquisition time. In the future, it is intended to extend to the 3-dimensional case (tomography) too.
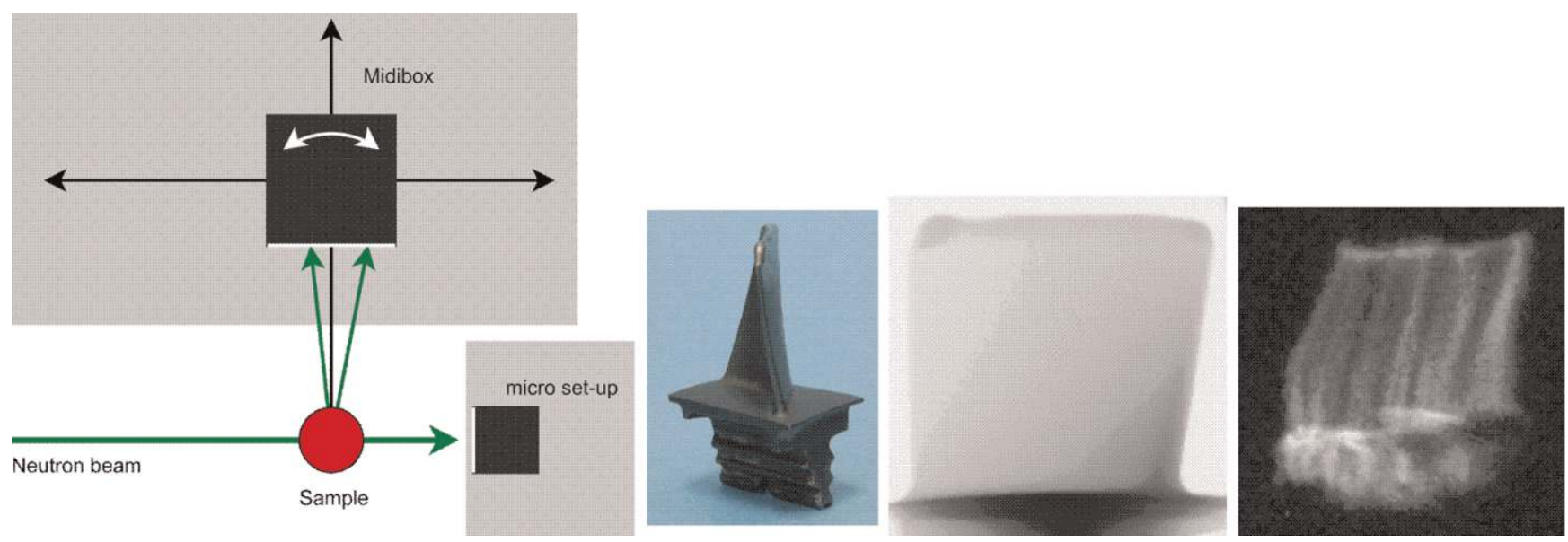

Fig. 7. Left: Schematic view onto the double detector setup where a sample is imaged in transmission mode and the diffracted neutrons are registered simultaneously aside the sample with a second imaging device; Right: "single crystal" turbine blade (photo, transmission image, diffracted image). 


\section{Phase based neutron imaging}

Neutrons are uncharged elementary particles which can be considered as waves according to de Broglie's relation $(\lambda=h / m v ; h=$ Planck's constant, $m=$ neutron mass, $v=$ neutron velocity). As such, the neutron wave gets refracted at material edges, which results in a sudden change in the beam distribution (intensity, direction).

The refractive index has been derived as:

$n=1-\delta=1-\frac{\lambda^{2} \cdot N \cdot b_{\mathrm{c}}}{2 \pi} \approx 1-10^{-6}$

Even if the deviation of $n$ from 1 is very small, how strongly the beam is deviated from its straight direction depends on the coherent scattering length $b_{\mathrm{c}}$ of the investigated material, its nuclear density $N$, but mainly on the neutron wavelength $\lambda$. The phase shift $\delta$ can be either positive (for most materials) or negative (e.g. Ti or Mn) in the case of neutrons.

In practical neutron imaging, refraction artifacts were often not seen because of the coarse detector resolution, the usage of mainly thermal neutrons and the inadequate sample-detector distances. Consequently, cold neutrons (3 to $10 \AA$ ) are obviously in favor when refraction should be visualized and used for material research.

These enhancement features (see Fig. 8) can be used to identify inner defects in material samples with dimensions below the detector resolution or contact areas of neighboring material with the same attenuation, but different phase effects.

However, if the edge enhancement is misleading in radiography or tomography studies, the samples have to be positioned closer to the detector or the experiment could be performed using thermal neutrons instead of cold ones.

Another methodical approach in phase contrast imaging the use of a grating interferometer as mentioned below (Section 9d).

\section{Further trends}

There are still many approaches to extend the usage of neutron imaging methods for materials research. The number of neutron imaging facilities suitable for advanced methods worldwide is still limited to about 15,5 of them were built within the last few years or underlay a major upgrade. In addition, 4 projects have been initiated at the pulsed neutron sources to build dedicated imaging facilities using the energy-resolution option in the time-of-flight mode. The potential is even larger if the operators of strong neutron sources decide to go ahead with neutron imaging activities. A data base prepared by IAEA and ISNR counts presently 43 facilities with various performances, not mentioning further activities in China, France, the Netherlands and Russia.

Currently, there are the following other developments of neutron imaging methods towards materials research applications with the potential for an extension to the third dimension as tomography tools:

a. Data fusion between neutron and X-ray imaging with a setup which allows pixel-conform acquisition. Both pixel matrices have to be combined and superimposed in order to derive synergetic knowledge about the sample material. Such a facility has been realized at the NEUTRA beam line as XTRA implementation. It depends very much on the investigated material combination what analytical algorithm has to be used to enhance and to superimpose the data in the right manner. Studies in this respect are still under development and improvement.

b. The perfect combination of diffraction and imaging as an extension of the double-detectors setup (see Section 7) will enable the description of spatially resolved structural data together with the volumetric distribution of the bulk material itself. This kind of installation is

materials at $30 \mathrm{~mm}$ distance, $20 \mathrm{~mm}$ aperture
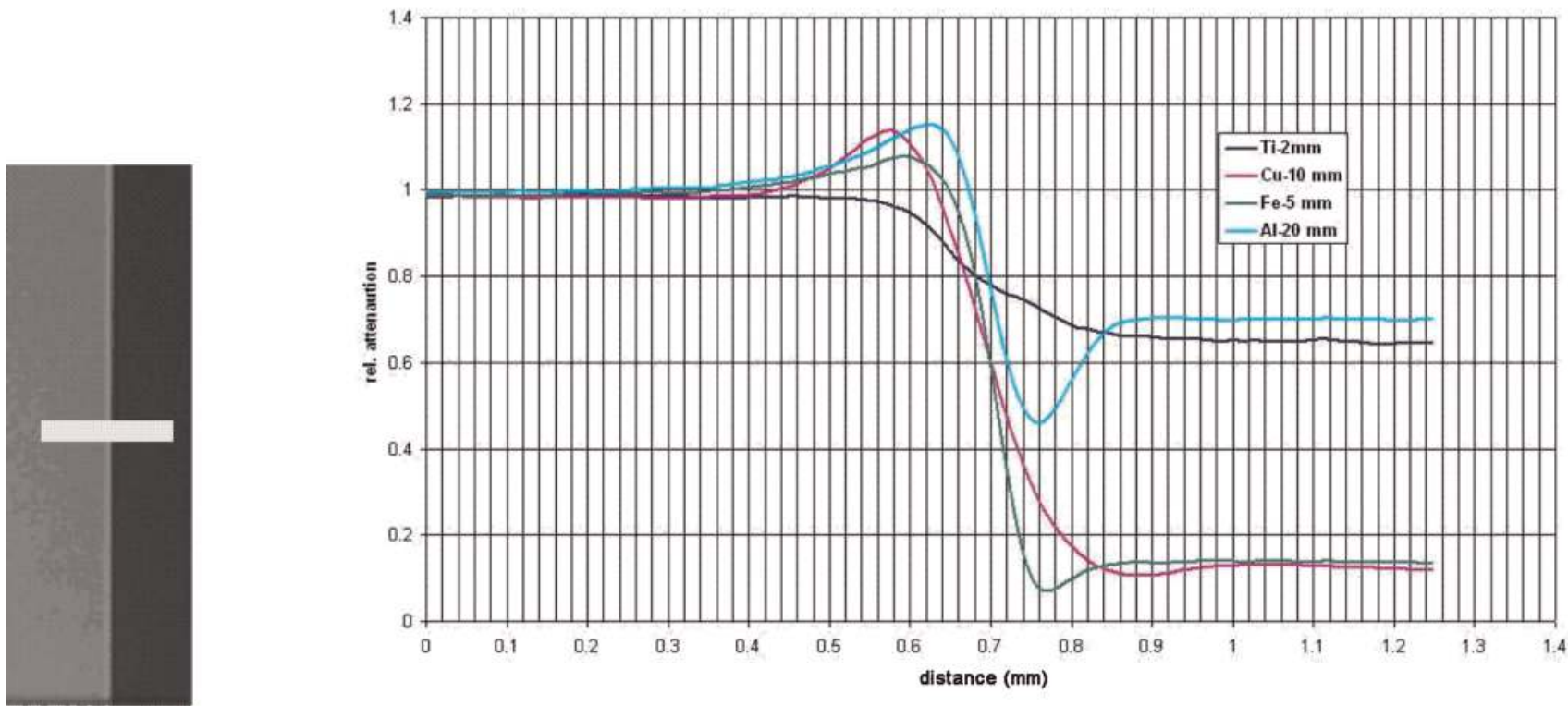

Fig. 8. Refraction effects at material edges: $20 \mathrm{~mm} \mathrm{Al}$ in the image left can produce effects at the edge as shown the diagram - conditions: cold neutrons; detector-sample-distance $30 \mathrm{~mm}$; beam aperture $20 \mathrm{~mm}$; right: profiles across the edges for different materials: Al, Fe, Cu have a positive phase shift and Ti a negative one. 
now foreseen at target station 2 at ISIS as the IMAT facility [22].

c. The use of epithermal neutrons (eV range) enables accessing resonances in the cross-sections of relevant materials $(\mathrm{U}, \mathrm{Ta}, \mathrm{I}, \ldots)$ which are measurable in bulk arrangements with a high sensitivity [28]. In particular, time-of-flight imaging methods with detection systems operating in a synchronized trigger mode can favorably be used for such studies. The upcoming imaging beam lines at pulsed spallation sources will take this option into account. The user potential is various, but nuclear materials can favorably be investigated and new fuel development programs can benefit.

d. Phase based investigations are also performed by using grating interferometer devices [29]. They allow deriving directly data for the phase shift $\delta$ (see Eq. (7)) as an independent material parameter. In addition, the socalled "dark-field image" can be obtained which is caused by small-angle scattering artifacts in the sample material or by magnetic phenomena. This technique is not directly realized with one single image, but the resulting phase contrast or the dark-field image has to be derived from suitable sequences of steps in the grating device.

\section{Conclusions}

We have given an overview of existing and future neutron imaging approaches for materials research on the basis of neutron interaction with the materials under investigation. The specific method can be chosen and adapted to the needs of the scientists or engineers involved and the composition of their samples.

Similar to X-ray tomography, there is a trend to higher spatial resolution, increased contrast and also time sequences in $2 \mathrm{D}$ and 3D. However, the problem of the limited neutron intensity at the existing neutron sources needs an optimization process for each individual neutron imaging application.

\section{References}

[1] J. Banhart (Ed.): Advanced tomographic methods in materials research and engineering, Oxford University Press, Oxford, (2008). DOI:10.1093/acprof:oso/9780199213245.001.0001

[2] B. Schillinger, E. Lehmann, P. Vontobel: Physica B: Condensed Matter, 276-278 (2000) 59-62.

[3] N. Kardjilov, F. Fiori, G. Giunta, A. Hilger, F. Rustichelli, M. Strobl, J. Banhart, R. Triolo: J. Neutron Res. 14 (2006) 29-36. DOI: 10.1080/10238160600673201.

[4] E.H. Lehmann, P. Vontobel, G. Frei, C. Brönnimann: Nucl. Instrum. Methods Phys. Res. A 531 (2004) 228-237. DOI:10.1016/j.nima.2004.06.010

[5] A. Tremsin, J. McPhate, J. Vallerga, O. Siegmund, J. Hull, B. Feller, E. Lehmann: Nucl. Instrum. Methods Phys. Res. A 604 (2009) 140. DOI:10.1016/j.nima.2009.01.041

[6] F. Natterer, F. Wübbeling: Mathematical Methods in Image Reconstruction, Soc. Ind. Appl. Math., Philadelphia (2001), ISBN 0-89871-472-9.

[7] http://www.ugct.ugent.be/software.php.

[8] A. Kaestner: Nucl. Instrum. Methods Phys. Res. A 651 (2011) 156-160. DOI:10.1016/j.nima.2011.01.129

[9] http://www.volumegraphics.com/en/products/vgstudio-max.html.

[10] http://www.paraview.org/.
[11] M. Holt : Annu. Rev. Mater Res. 43 (2013) 183-211. DOI:10.1146/annurev-matsci-071312-121654

[12] E.H. Lehmann, P. Vontobel, L. Wiezel: Non-Destr. Test. Eval. 16 (1997) 191-202. DOI:10.1080/10589750108953075

[13] A.P. Kaestner, S. Hartmann, G. Kühne, G. Frei, C. Grünzweig, L. Josic, F. Schmid, E.H. Lehmann: Nucl. Instrum. Methods Phys. Res. A 659 (2011) 387-393. DOI: 10.1016/j.nima.2011.08.022

[14] R. van Lang: Technical Studies of Renaissance Bronzes, Diss. TU Delft, (2012), ISBN 978-90-71450-49-5.

[15] http://www.telegraph.co.uk/earth/environment/archaeology/ 9991936/Mary-Rose-reveals-armour-piercing-cannonballsecret.html.

[16] E.H. Lehmann, G. Frei, G. Kühne, P. Boillat: Nucl. Instrum. Methods Phys. Res. A 576 (2007) 389-396. DOI:10.1016/j.nima.2007.03.017

[17] http://www.psi.ch/sinq/.

[18] J. Granada: Naturforsch. 39a (1987) 1160-1167.

[19] W. Kockelmann, G. Frei, E.H. Lehmann, P. Vontobel, J. Santisteban: Nucl. Instrum. Methods Phys. Res. A 578 (2007) 421-434. DOI:10.1016/j.nima.2007.05.207

[20] F. Salvemini, F. Grazzi, S. Peetermans, F. Civita, R. Franci, S. Hartmann, E. Lehmann, M. Zoppi: J. Anal. At. Spectrom. 27 (2012) 1494-1501. DOI:10.1039/c2ja30035d

[21] E. Lehmann, G. Frei, P. Vontobel, L. Josic, N. Kardjilov, A. Hilger, W. Kockelmann, A. Steuwer: Nucl. Instrum. Methods Phys. Res. A 603 (2009) 429. DOI:10.1016/j.nima.2009.02.034

[22] W. Kockelmann S.Y. Zhanga, J.F. Kelleher, J.B. Nightingale, G. Burca, J.A. James: Physics Procedia 43 (2013) 100-110. DOI:10.1016/j.phpro.2013.03.013

[23] Y. Kijanagy: ERNIS beam line for neutron imaging at JPARC, private communication.

[24] A.V. Belushkin: Neutron News 2(2) (1991) 14-18. DOI: $10.1080 / 10448639108218724$

[25] H. Bilheux: VENUS project at SNS, Oak Ridge National Laboratory, private communication.

[26] M. Strobl: The ODIN project at ESS Lund, private communication.

[27] A. Kaestner, B. Münch, L. Butler, P. Trtik: Opt. Eng. 50 (2011) 123-201. DOI: $10.1117 / 1.3660298$

[28] A. Tremsin, J.B. McPhate, J.V. Vallerga, O.H.W. Siegmund, W. Kockelmann, E.M. Schooneveld, N.J. Rhodes, W.B. Feller: IEEE Trans. Nucl. Sci. 59 (2012) 3272-3277. DOI:10.1109/TNS. 2012.2215627

[29] F. Pfeiffer, C. Grünzweig, O. Bunk, G. Frei, E. Lehmann, C. David: Phys. Rev. Lett. 96 (2006) 215505. DOI:10.1103/PhysRevLett.96.215505

(Received August 30, 2013; accepted January 15, 2014; online since February 26, 2014)

\section{Correspondence address}

Dr. Eberhard H. Lehmann

Paul Scherrer Institut

CH-5232 Villigen PSI

Switzerland

Tel: +41563102963

Fax: +41563103131

E-mail: eberhard.lehmann@psi.ch

\section{Bibliography}

DOI $10.3139 / 146.111053$

Int. J. Mater. Res. (formerly Z. Metallkd.)

105 (2014) 7; page 664-670

(C) Carl Hanser Verlag GmbH \& Co. KG

ISSN 1862-5282 